\title{
Intensive occlusion therapy for amblyopia
}

\author{
Suzanne E Dorey, Gillian G W Adams, John P Lee, John J Sloper
}

\begin{abstract}
Aim-To study the effects of supervised inpatient occlusion treatment for amblyopia in children who had failed to respond to outpatient treatment.

Methods-A retrospective study of 39 children admitted to a paediatric ophthalmic ward for 5 days of supervised intensive occlusion therapy having previously failed to respond to outpatient occlusion. Visual acuity of amblyopic and fellow eyes was recorded at each clinic visit before admission, daily during admission, and at each outpatient visit after discharge.

Results-There was no significant overall improvement in visual acuity during a mean of 9 months of attempted outpatient occlusion before admission. During the 5 days of admission 26 children (67\%) gained at least one line of acuity in their amblyopic eye and five (13\%) gained three or more lines (mean gain 1.03 Snellen lines). The acuities of both the amblyopic and fellow eyes subsequently improved with continuing part time patching as an outpatient, including in nine of the children who did not respond during admission. At the last recorded visit, at a median time of 14 months after discharge, $13(33 \%)$ of the patients had an acuity of at least $6 / 12$ in their amblyopic eye.

Conclusions-The acuity of amblyopic eyes did not improve without effective treatment. Subsequent supervised inpatient occlusion therapy was effective in the majority of the children.

(Br F Ophthalmol 2001;85:310-313)
\end{abstract}

Amblyopia is a reduced corrected visual acuity without evidence of organic eye disease. It is caused by abnormal visual experience during a sensitive period of visual development in early childhood, most commonly from a squint or from visual deprivation caused by unequal refractive errors. It affects up to $3.5 \%$ of children and is the most common cause of uniocular visual impairment in children and young adults. ${ }^{1}$ It has been treated by correction of refractive errors followed by occlusion of the fellow eye for many years with apparent success provided that amblyopia has been detected before completion of the critical period of visual development. ${ }^{2-6}$

Recently, the effectiveness of occlusion treatment for amblyopia has been questioned because it has not been subjected to formal controlled trials and it has been suggested that much of the improvement in acuity seen is spontaneous and unrelated to occlusion. ${ }^{7}$ Following on from this the rationale for vision screening of children has been questioned. ${ }^{7}$ However, many clinicians have been concerned about randomisation of amblyopic children to a "no treatment" group in a controlled trial because from their clinical experience this means depriving children of an effective treatment. ${ }^{89}$ One approach to this dilemma has been to compare outcomes in children who have not complied with treatment with those who have. ${ }^{8}$ Although useful, this approach does not compare equivalent groups with and without patching because of differences between those children who comply and those who do not. It also does not demonstrate that the children who did not comply had the potential to respond to patching.

We offer to admit children who have failed to respond to occlusion therapy, usually because of poor compliance, for supervised inpatient occlusion. We therefore have a group of children in whom initial outpatient therapy had failed, mainly from poor compliance, and who have then undergone a short period of supervised inpatient occlusion where compliance was directly observed. Once compliance had been established the majority of parents reported that their children continued to wear their patches at home. This paper describes retrospective clinical results on such patients. Each child acts as its own control and so these data resemble a natural crossover trial of treatment following no treatment in a group of children who have proved the most resistant to amblyopia therapy. There are significant limitations to this approach and it would be preferable from a purely scientific viewpoint to do such a trial prospectively. However, this would involve deliberately withholding from children what is believed by many to be an effective treatment whereas this approach does not.

A preliminary account of these data was presented at the 25th meeting of the European Strabismological Association. ${ }^{10}$

\section{Patients and methods}

The case records of 39 children from the 41 who had been admitted to the paediatric ward of Moorfields Eye Hospital for intensive, supervised occlusion therapy from January 1995 to December 1996 were analysed. In all cases there was documented evidence of failure to respond adequately to outpatient occlusion. Appropriate spectacles had been prescribed. An initial occlusion regime of 2 hours a day had been prescribed and increased if the vision failed to respond. If the child refused to comply with occlusion then atropine penalisation was considered and had been tried without success in seven cases $(21 \%)$. Ten patients had been referred for a second opinion having failed to respond to treatment in other units. In $76 \%$ of
Accepted for publication 22 September 2000 
cases the parents admitted to problems of compliance with occlusion treatment and often also with spectacle wear. Although the unreliability of parental reporting of compliance is well described, ${ }^{11}$ there seems little doubt that compliance was very poor in these children before admission. Three children were admitted who had failed to improve as outpatients, although their compliance had been thought to be good.

Visual acuity was recorded using age appropriate methods of assessment. In the youngest children this was by Cardiff cards or Kays pictures. In most children Sheridan-Gardiner single letter matching was used, with some progressing to a linear Snellen acuity. Visual

Table 1 Mean $\log M A R$ visual acuities of 39 amblyopic and fellow eyes before, during, and after admission for occlusion

\begin{tabular}{lll}
\hline & \multicolumn{2}{l}{ Mean logMAR equivalent acuity } \\
\cline { 2 - 3 } & $\begin{array}{l}\text { Amblyopic eye } \\
\text { Mean (SD) }\end{array}$ & $\begin{array}{l}\text { Fellow eye } \\
\text { Mean (SD) }\end{array}$ \\
\hline Initial acuity & $1.10(0.14)$ & $0.39(0.41)$ \\
Acuity on admission & $1.02(0.20)$ & $0.29(0.32)$ \\
Significance of difference from initial acuity & $\mathrm{p}=0.10$ & $\mathrm{p}=0.09$ \\
Acuity on discharge & $0.82(0.34)$ & $0.28(0.35)$ \\
Significance of difference from acuity on admission & $\mathrm{p}=<0.001$ & $\mathrm{p}=0.77$ \\
Acuity 3 months after discharge & $0.68(0.44)$ & $0.18(0.19)$ \\
Final acuity & $0.63(0.11)$ & $0.13(0.17)$ \\
Significance of difference from acuity on discharge & $\mathrm{p}<0.01$ & $\mathrm{p}<0.002$ \\
\hline
\end{tabular}

$\star$ Paired $t$ test.

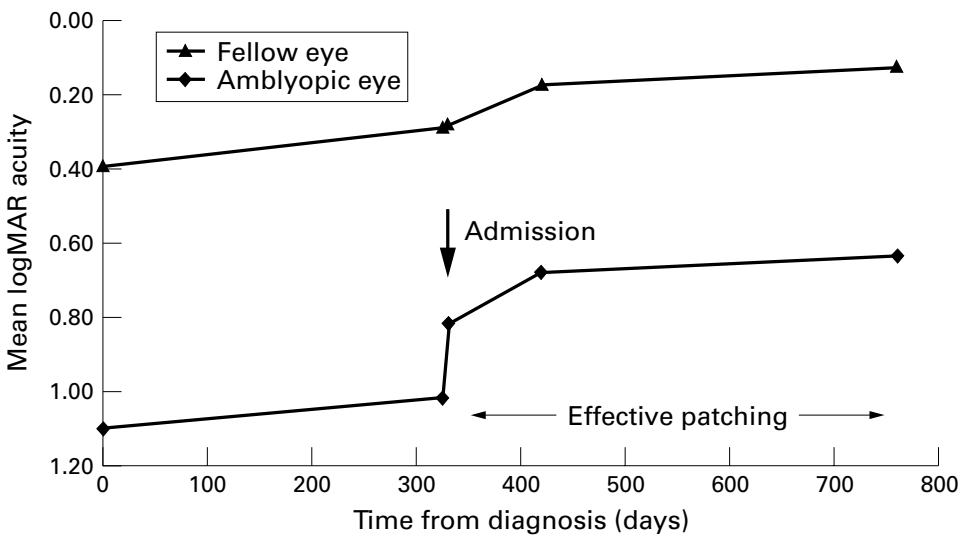

Figure 1 Mean $\log M A R$ equivalent acuity for the amblyopic and fellow eyes of 39 children before, during, and after admission for inpatient occlusion. The intervals from first visit to admission and from discharge to final visit are mean values.

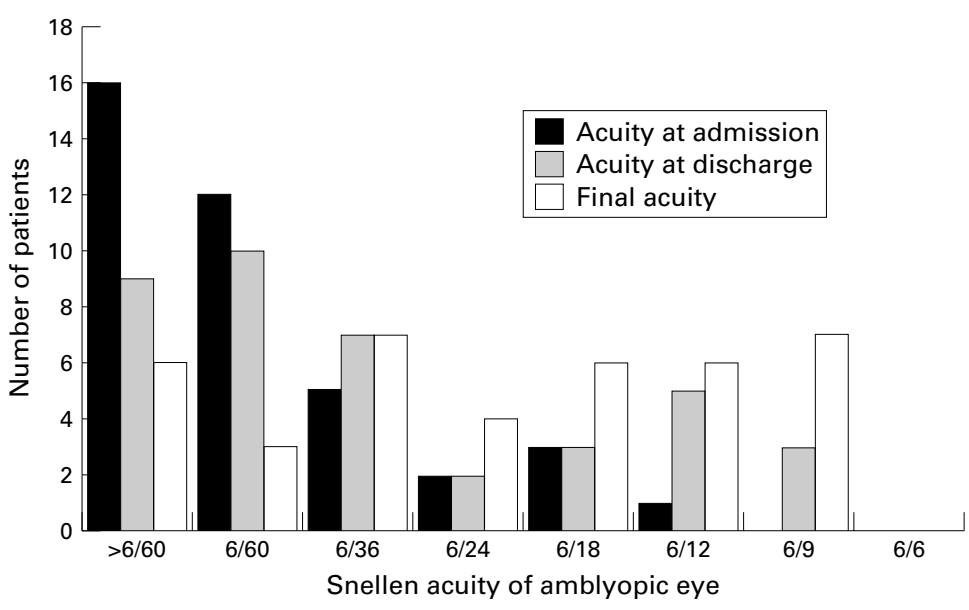

Figure 2 Distribution of the Snellen acuities of the amblyopic eye of 39 children at admission, discharge, and final visit. acuities have been recorded as Snellen equivalents and lines of improvement in acuity are equivalent to lines on the Snellen chart. For statistical analysis and Figure 2 acuities have been converted to $\log$ MAR equivalents. ${ }^{12}$ Comparisons have been performed using paired $t$ tests. Analysis was also performed using non-parametric methods on the unconverted data and gave very similar results.

Visual acuity was recorded at each outpatient visit, on admission, and daily during admission. During admission the children and a parent spent 5 days in hospital receiving full time occlusion therapy and appropriate spectacle wear under direct staff supervision. During admission they were seen daily, usually by the same orthoptist and visual acuity was measured by the same method. In the majority of children this was by Sheridan-Gardiner single letter matching. After discharge, vision was checked at 1 week, 3 months, 6 months, and at the most recent clinic visit if still receiving treatment, or at the final clinic visit if discharged.

\section{Results}

The average age at presentation to Moorfields Eye Hospital was 2 years 4 months (range 2 months to 5 years), with an eye problem that had first been suspected at an average age of 9 months (range birth to 4 years). Home supervised occlusion was attempted for a mean of 9 months before admission (range 1 week to 3 years). Thirty five children $(89 \%)$ were also prescribed glasses to be worn full time. The commonest cause of amblyopia was a convergent squint in isolation (18 patients) or associated with anisometropia (10 patients). Three patients had a divergent squint and one had anisometropia alone. Amblyopia was associated with a structural abnormality in seven cases - two with congenital cataract, two with cranial nerve palsies, two with a lid haemangioma, and one associated with a coloboma.

Between the first visit, when home supervised occlusion was started and the time of admission, there was no significant improvement in the mean visual acuity of the amblyopic eye (Table 1; Fig 1). Twenty one children $(62 \%)$ were documented as having no improvement or a worsening of acuity and in a further five children the vision was so poor that there was no cooperation for acuity assessment in the amblyopic eye. From the whole group only five children had gained more than one line before admission and only three had an acuity in the amblyopic eye of better than $6 / 36$. Visual acuities at the time of admission are shown in Figure 2.

The average age at admission was 3.2 years (range 10 months to 6.5 years). During the 5 days of admission 26 of the children (67\%) showed an improvement of one or more lines of acuity with five $(13 \%)$ gaining three or more lines. The overall mean improvement in the acuity of the amblyopic eye (Table 1; Fig 1) corresponded to approximately one line on the Snellen chart. Although the largest improvements in acuity were seen in children aged 4 
and under, there was no significant overall difference in the response to patching with age. There was no significant change in the acuity of the fellow eye during admission (Fig 1; Table 1). The distributions of acuity for the amblyopic eyes at admission, discharge, and the final visit are shown in Figure 2.

After discharge the acuity in the amblyopic eye continued to improve with outpatient occlusion. Follow up was for an average of 19 months (range 4 months to 3.5 years), with the mean age at last assessment of 4.8 years. Twenty three $(58 \%)$ of the patients gained one or more lines of acuity after discharge, nine $(23 \%)$ were stable, and seven $(18 \%)$ lost one or more lines of vision, probably because of poor compliance. Of the 13 children whose amblyopic eye failed to improve during the 5 days of admission nine subsequently gained one or more lines and four gained three or more (Fig 3). The amblyopic eyes of these children improved more following discharge from hospital than those of children who responded during admission (Fig 3). The numbers of lines of acuity gained by the amblyopic eye of each child from admission to the last outpatient visit are shown in Figure 4. At the last

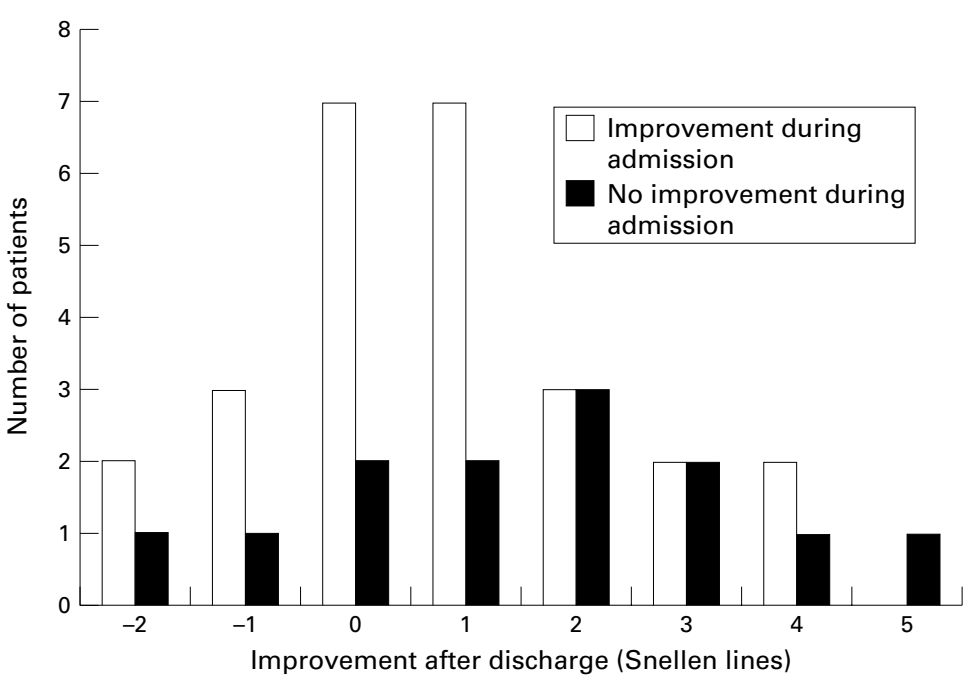

Figure 3 Comparison of subsequent improvement in the acuity of the amblyopic eye following discharge in children who did or did not improve during admission. Children who did not improve during admission showed a larger subsequent improvement.

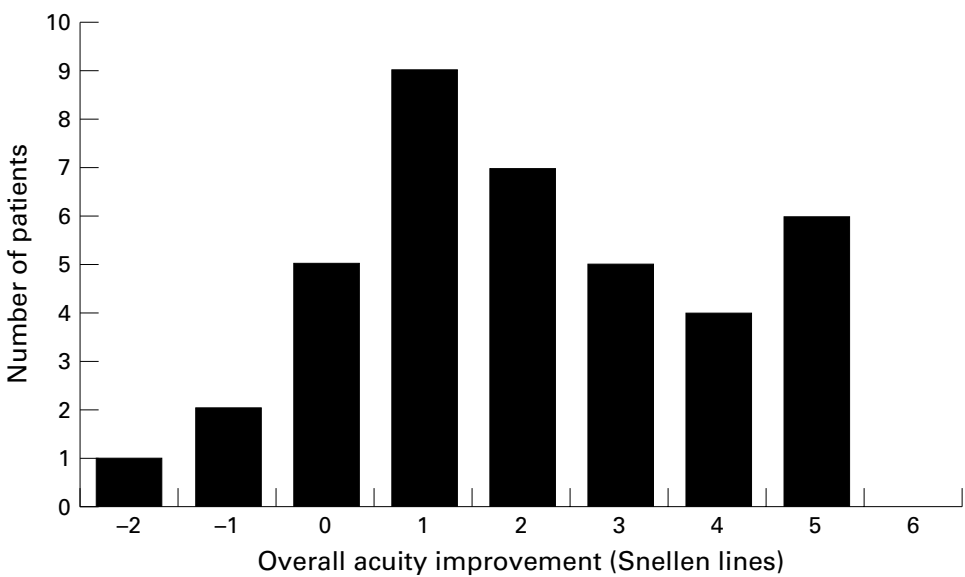

Figure 4 Number of Snellen lines of acuity gained by the amblyopic eye between admission and final visit for 39 children. recorded visit 13 children (33\%) had an acuity of at least $6 / 12$ in their amblyopic eye. There was also a significant improvement in the acuity of the fellow eye in the follow up period after discharge (Fig 1; Table 1).

\section{Discussion}

This study has demonstrated that the acuity of the amblyopic eye did not improve spontaneously over a mean of 9 months in 39 children, most of whom complied very poorly with patching treatment during this time. When they did comply with treatment their acuity improved rapidly. The fact that there was no improvement in the acuity of the amblyopic eye over a number of clinic visits during the preadmission phase and that no improvement occurred in the fellow eye during the period of admission makes it very unlikely that a learning effect accounts for this improvement. Both the amblyopic and fellow eyes improved with continuing part time patching after admission making a detrimental effect of patching on acuity of the fellow eye also very unlikely.

This study has some limitations stemming from the fact that it was a retrospective study of clinical practice. In particular, visual acuity was not necessarily measured by the same methods throughout because of the changing age of the children. However, the methods used to measure acuity in older children are in general more demanding and would be expected to reduce measured acuity and minimise any improvements observed. In particular, measurements made during the period of admission, when the most striking improvement occurred, were all done using the same method and in most instances were performed by the same orthoptist for individual children. Although test-retest reliability was not formally assessed, the acuity of the amblyopic eye generally remained stable or improved progressively through the week of admission, with few apparently random changes. There are also limitations of analysis imposed by the use of Snellen based rather than $\log$ MAR acuity measures. ${ }^{12}$ The acuity data were transformed into $\log M A R$ equivalents for statistical analysis but the statistical conclusions were also confirmed using nonparametric statistical methods on the untransformed data. The comparisons of acuity are within subject, making them more reliable, and the effects found were robust.

The mean improvement of the amblyopic eye during admission was one Snellen line of acuity, which may seem modest. One third of the children did not respond to occlusion during the 5 days of admission which dilutes the mean effect of treatment. Of these 13, nine subsequently improved and the improvement following discharge in these children was greater than that in the children who improved during admission. Once compliance with patching had been established, the majority of parents reported continuing compliance and it seems likely that the response to patching was simply slower in some of these children. Overall, of the 39 children, 35 gained at least one line of acuity, and 16 gained two or more lines with patching. Five children who had improved 
during admission subsequently lost one or two lines of acuity. Although compliance was not directly monitored, several children were known to have missed follow up appointments and to have continuing problems with compliance. A number of children were also known to have organic ocular defects, such as optic nerve hypoplasia, and occlusion was being performed because it was not clear how much acuity was limited by the organic problem and how much by amblyopia. By the final assessment the acuity in 15 of the children (38\%) improved by at least three lines and, overall, 13 (33\%) children who had failed to respond to outpatient occlusion finished with an acuity of 6/12 or better in their amblyopic eye. This would probably be sufficient to allow them to continue driving if the vision of their good eye was lost through injury or disease and represents a very worthwhile gain. At the time of admission 16 of the children would probably have required blind registration and a further 17 partial sight registration if they had lost the vision in their good eye as an adult. Following treatment only six would be likely to require blind registration and 11 partial sight registration under the same circumstances.

A recent NHS review suggested that there was insufficient evidence that occlusion therapy for amblyopia was effective. ${ }^{7}$ It further implied that as this was the case there was little point in continuing vision screening in children, as the target disease process was amblyopia for which treatment was not proved to be effective. This caused concern to many dealing with children's vision screening and treatment, whose the clinical experience is that occlusion therapy is effective in most children provided that they comply with treatment. The present study has provided evidence both that amblyopia does not, in general, improve spontaneously and that patching is an effective treatment even in the majority of those children who are most resistant to therapy.

Proprietary interests: None.

Grant support: None.

1 Von Noorden GK. Binocular vision and ocular motility. 5th ed. St Louis: Mosby, 1995

2 Eppelbaum M, Milleret C, Buisseret P, et al. The sensitive period for strabismic amblyopia in humans. Ophthalmology 1993;100:323-7.

3 Garzia RP. Efficacy of vision therapy in amblyopia: a literature review. Am f Optom Physiol Optics 1987;64:393-404.

4 Lithander J, Sjostrand J. Anisometropic and strabismic amblyopia in the age group 2 years and above: a prospective amblyopia in the age group 2 years and above: a prospective
study of the results of treatment. Br f Ophthalmol 1991;75: study of the $111-16$.

5 Hiscox F, Strong M, Thompson JR, et al. Occlusion for amblyopia; a comprehensive survey of outcome. Eye 1992; 6:300-9.

6 Woodruff G, Hiscox F, Thompson JR, et al. Factors affecting the outcome of children treated for amblyopia. Eye 1994;8: 627-31.

7 Snowdon SK, Stewart-Brown S. Preschool vision screening: reviews and dissemination. Report 9, 1997 .

8 Simons K, Preslan M. Natural history of amblyopia untreated owing to lack of compliance. $\mathrm{Br} \mathcal{F}$ Ophthalmol 1999;83:582-7.

9 Lee J, Adams G, Sloper J, McIntyre A. Future of preschool vision screening. Cost effectiveness of screening for vision screening. Cost effectiveness of screening for

10 Dorey SE, Adams GGW, Lee JP. Intensive occlusion therapy for amblyopia. Proceedings of the 25th Meeting of the for amblyopia. Proceedings of the 25th Meeting of the 25-30.

11 Fielder AR, Irwin M, Auld R, et al. Compliance in amblyopia therapy: objective monitoring of occlusion. $\mathrm{Br} f$ Ophthalmol 1995;79:585-9.

12 Moseley MJ. Graphical representation of visual acuity data. Ophthalmic Physiol Opt 1997;17:441-2. 